\title{
On Group Von Neumann Algebras with Vector-Valued Functions
}

\author{
Essé Julien ATTO* and V.S. Kofi ASSIAMOUA** \\ Department of Mathematics - FaST, University of Kara, BP 404 Kara-Togo* \\ Department of Mathematics - FDS, University of Lomé, BP 1515 Lomé,-Togo** \\ E-mail : attoej@yahoo.fr
}

\begin{abstract}
Let $G$ be a locally compact group equipped with a normalized Haar measure $\mu, A(G)$ the Fourier algebra of $G$ and $V N(G)$ the von Neumann algebra generated by the left regular representation $\lambda$ of $G$. In this paper, we introduce the space $\operatorname{VN}(G, \mathcal{A})$ associated with the Fourier algebra $A(G, \mathcal{A})$ for vector-valued functions on $G$, where $\mathcal{A}$ is a $H^{*}$-algebra. Some basic properties are discussed in the category of Banach space, and also in the category of operator space.
\end{abstract}

Keywords: Compact groups, Fourier algebra, Group von Neumann algebra, Operator spaces.

2010 Mathematics Subject Classification: Primary: 22D15; Secondary: 46E40, 46J10, 43A30, 46 L07.

Language: English

Date of Submission: 2018-03-31

Date of Acceptance: 2018-04-20

Date of Publication: 2018-04-30

ISSN:2347-1921

Volume: 14 Issue: 01

Journal: Journal of Advances in Mathematics

Website: https://cirworld.com

This work is licensed under a Creative Commons Attribution 4.0 International License. 


\section{Introduction}

The theory of rings of operators called today von Neumann algebra was first introduced and developed by Murray and von Neumann in 1936 [10], with the aim of developing a suitable mathematical framework for quantum mechanics. Today, it extends into the larger theory of noncommutative geometry and intervenes in various fields such as the theory of representations and the $L_{2}$-invariants theory.

In mathematics, one can assign to a locally compact group $G$ an operator algebra such that representations of the algebra are related to representations of the group. Any space constructed in this way is called group algebra.

Let $L_{p}(G)(1 \leq p<\infty)$ be the set of all functions $f: G \rightarrow \mathbb{C}$, such that $\int_{G}|f(x)|^{p}<\infty$, and $C(G)$ the set of all continuous complex-valued functions on $G$. These spaces form Banach algebras under usual operations and convolution. In [13], D. Z. Spicer extended the group algebras $L_{p}(G)$ and $C(G)$ to group algebras of vectorvalued functions respectively denoted $B_{p}(G, A)$ and $C(G, A)$. Mainly, $B_{p}(G, A)$ is the space of all continuous functions $f: G \rightarrow A$ such that $\int_{G}\|f(x)\|_{A}^{p} d x<\infty$ (usually denoted $L_{p}(\mathrm{G}, \mathrm{A})$ )), and $C(G, A)$ is the space of all continuous functions from $G$ to $A$, where $A$ is a Banach algebra.

As far as we know, the space $V N(G)$ is associated with the space of complex-valued continuous functions on $G$ with compact support and there is no analog for vector-valued functions yet. In this paper, we want to extend this definition in the case of Banach algebra-valued functions with additional conditions.

Section 2 deals with preliminaries.

In Section 3, we introduce a vector-valued analog of the group $C^{*}$-algebra $C^{*}(G)$ and the reduced group $C^{*}$ algebra $C_{r}^{*}(G)$ which will be denoted respectively by $C^{*}(\mathrm{G}, \mathcal{A})$ and $C_{r}^{*}(\mathrm{G}, \mathcal{A})$ where $\mathcal{A}$ is assumed to be an $H^{*}$ algebra.

Now, we deal with one of the main results of our paper in Section 4: the generalization of the space $V(G)$ in the case of vector-valued functions. The vector-valued von Neumann algebra $\operatorname{VN}(G, \mathcal{A})$ is the weak operator topology closure of $C^{*}(G, \mathcal{A})$. We discuss some basic properties of this space.

Finally, in Section 5, the spaces $V N(G)($ resp. $\mathrm{A}(G))$ and $V N(G, \mathcal{A})(\operatorname{resp} . \mathrm{A}(\mathrm{G}, \mathcal{A}))$ ) are equipped with their natural operator space structure. We then study some properties of isomorphisms and isometries in the category of operator spaces. A characterization of completely bounded multiplier on a specific dense subspace of by $A(G, \mathcal{A})$ is established.

\section{Preliminaries}

In this section, we recall some notations and results related to locally compact groups and operator spaces. The reader is referred to P. Eymard [6], Effros and Ruan [5] for more details. Through this paper, we shall assume that $G$ is a locally compact Hausdorff topological group endowed with its left Haar measure $\mu$ normalized so that $\mu(G)=1$.

Let $B(G)$ be the Fourier-Stieltjes algebra of $G$, then the Fourier algebra $A(G)$ is defined as the Banach subalgebra of $B(G)$ generated by the continuous functions of positive type with compact support. $A(G)$ is identified with the space

$$
\left\{f * \tilde{\mathrm{g}}: f, g \in L_{2}(G)\right\}(\text { see Eymard [6]) }
$$

where $f * g(s)=\int_{\mathrm{G}} f\left(s t^{-1}\right) g(t) d t$ is the convolution product and $\tilde{f}: t \mapsto \overline{f\left(t^{-1}\right)}$.

$A(G)$ is equipped with the norm 


$$
\|u\|_{A(G)}=\inf _{u=f * g}\|f\|_{L_{2}(G)}\|g\|_{L_{2}(G)}
$$

and is known to be a subalgebra of $C_{0}(G)$ (the space of decreasing continuous functions on $G$, vanishing at infinity), so a commutative Banach algebra with respect to the pointwise multiplication.

Let $C_{c}(G)$ be the space of complex-valued continuous functions on $G$ with compact support; this acts on $L_{2}(G)$ by left convolution, and forms a *-subalgebra of $\operatorname{Hom}\left(L_{2}(G)\right):\left\{\Lambda_{f}: L_{2}(G) \ni g \mapsto f * g \in L_{2}(G), f \in C_{c}(G)\right\}$ ， , which closure is $C_{r}^{*}(G)$, the reduced group $C^{*}$-algebra. The group $C^{*}$-algebra $C^{*}(G)$ is obtained by taking the supremum over all $C^{*}$-norms. The weak operator topology closure of $C_{r}^{*}(G)$ is called the group von Neumann algebra of $G$, denoted $V N(G)$. Equivalently, if $\mathcal{B}\left(L_{2}(G)\right)$ denotes the space of all bounded linear maps on $L_{2}(G)$, we have:

$$
V N(G)=\{\lambda(s): s \in G\}
$$

where $\lambda: G \rightarrow\left(\mathcal{B} L_{2}(G)\right)$ is the left regular representation of $G$.

$$
\begin{aligned}
& \lambda(s): L_{2}(G) \quad \rightarrow \quad L_{2}(G) \\
& f \quad \mapsto g, g(t)=f\left(s^{-1} t\right) .
\end{aligned}
$$

$A(G)$ is the predual of $V N(G)$.

An operator space is a closed subspace of the space $\mathcal{B}(H)$ of all bounded operators on a Hilbert space $H$. In other words, it is a Banach space given together with an isometric linear embedding into the space $\mathcal{B}(H)$. An abstract characterization of operator spaces was given by Ruan in [5]. A complex vector space $E$ is an operator space if and only if for each integer $n \geq 1$, there is a complete norm $\|\cdot\|_{n}$ on $M_{n}(E)$, the space of $n \times n$ matrices with entries in $E$, such that the following properties are satisfied:

$$
\forall u \in M_{n}(E), v \in M_{m}(E), \alpha, \beta \in M_{n}
$$

(i) $\|u \oplus v\|_{n+m}=\max \left\{\|u\|_{n},\|v\|_{m}\right\}_{1}$

(ii) $\|\alpha u \beta\|_{n} \leq \alpha\|u\|_{n} \beta$.

A linear map $\phi: E_{1} \subset B\left(H_{1}\right) \rightarrow E_{2} \subset B\left(H_{2}\right)$ between two operator spaces is said to be completely bounded (c.b. in short) if the linear maps

$$
\begin{array}{llc}
\phi_{n}: M_{n}\left(E_{1}\right) & \rightarrow & M_{n}\left(E_{2}\right) \\
\left(a_{i j}\right)_{1 \leq i, j \leq n} & \mapsto\left(\phi\left(a_{i j}\right)\right)_{1 \leq i, j \leq n}
\end{array}
$$

are such that $\sup _{n \geq 1}\left\|\phi_{n}\right\|<\infty$. The completely bounded norm is denoted by $\|\phi\|_{c b}=\sup _{n \geq 1}\left\|\phi_{n}\right\|$. The space of all completely bounded maps from $E_{1}$ into $E_{2}$ is denoted $c b\left(E_{1}, E_{2}\right)$ and simply $c b\left(E_{1}\right)$ if $E_{1}=E_{2}$.

We give the following definition about $H^{*}$-algebras as introduced by Ambrose in [1]:

An involutive Banach algebra $\mathcal{A}$ over $\mathbb{C}$ with involution

$$
\begin{aligned}
& \text { * : } \mathcal{A} \rightarrow \mathcal{A} \\
& x \mapsto x^{*}
\end{aligned}
$$

is called an $H^{*}$-algebra if $\mathcal{A}$ admits an inner product $(\cdot$,$) satisfying the following postulates:$

(i) The underlying Banach space of $\mathcal{A}$ is a Hilbert space (of arbitrary dimension); 
(ii) For each $x \in \mathcal{A}$, there is an element in $\mathcal{A}$ denoted by $x^{*}$ and called an adjoint of $x$, such that for all $y, z \in \mathcal{A}$, we have both $(x y, z)=\left(y, x^{*} z\right)$ and $(y x, z)=\left(y, z x^{*}\right)$.

\section{The generalized group $C^{*}$-algebras $C_{r}^{*}(G, \mathcal{A})$ and $C^{*}(G, \mathcal{A})$}

In the sequel, $\mathcal{A}$ will denote an $H^{*}$-algebra and $G$ a compact topological group with Haar measure $\mu$, normalized so that $\mu(G)=1$. For $1 \leq p<\infty, L_{p}(G, \mathcal{A})$ is the space of all equivalence classes (modulo null functions) of all measurable functions $f: G \rightarrow \mathcal{A}$ such that $\int_{G}\|f(x)\|_{\mathcal{A}}^{p} d \mu(x)<\infty$, and $C_{c}(G, \mathcal{A})$ will denote the space of all continuous functions from $G$ to $\mathcal{A}$ with compactly support. The space $L_{p}(G, \mathcal{A})$ (resp. $C_{c}(G, \mathcal{A})$ ) equipped with the norm $\|f\|_{p}=\|f(x)\|_{\mathcal{A}}^{p} d \mu(x)\left(\right.$ resp. $\left.\|f\|_{\infty}=\sup _{x \in G}\|f(x)\|_{\mathcal{A}}\right)$ is a Banach space.

The Fourier algebra $A(G, \mathcal{A})$ on $G$ associated with functions $f: G \rightarrow \mathcal{A}$ is defined as the usual one:

$$
A(G, \mathcal{A}):=\left\{f * \tilde{g}: f, g \in L_{2}(G, \mathcal{A})\right\},
$$

where $\tilde{\mathrm{f}}(t)=\left(f\left(t^{-1}\right)\right)^{*_{\mathcal{A}}}$ and $*_{\mathcal{A}}$ is the involution in $\mathcal{A}$. Equipped with the norm

$$
\|u\|_{A(G, \mathcal{A})}:=\inf _{u=f * g}\left\{\|f\|_{2}\|g\|_{2}: f, g \in L_{2}(G, \mathcal{A})\right\}_{1}
$$

it becomes a Banach space.

If we set $f_{\mathrm{V}}(t)=f\left(t^{-1}\right)$, then $\tilde{f}(t)=\left(f_{\mathrm{V}}(t)\right)^{* \mathcal{A}}$.

The completion of $C_{c}(G, \mathcal{A})$ in the $L_{1}(G, \mathcal{A})$-norm is isomorphic to the space $L_{1}(G, \mathcal{A})$.

In this section we will generalize the group algebras $C_{r}^{*}(G)$ and $C^{*}(G)$ of complex-valued functions to those of vector-valued functions denoted $C_{r}^{*}(G, \mathcal{A})$ and $C^{*}(G, \mathcal{A})$, then we will study some of their properties. Recall that since $\mathcal{A}$ is an $H^{*}$-algebra, so is $L_{2}(G, \mathcal{A})$. Set $\langle,\rangle_{L_{2}}\left(\right.$ resp. $\left.\langle,\rangle_{\mathcal{A}}\right)$ the inner product associated with $L_{2}(G, \mathcal{A})$ (resp. with $\mathcal{A}$ ) as a Hilbert space. We have:

$$
\langle g, h\rangle_{L_{2}}=\int_{G}\langle g(x), h(x)\rangle_{\mathcal{A}} d x
$$

Proposition 3.1 Let $G$ be a locally compact group and $\mathcal{A}$ be an $H^{*}$-algebra.

(i) The space $C_{c}(G, \mathcal{A})$ acts boundedly on on $L_{2}(G, \mathcal{A})$ by left convolution.

(ii) The space $\mathcal{T}(G, \mathcal{A})=\left\{\Lambda_{f}: L_{2}(G, \mathcal{A}) \rightarrow L_{2}(G, \mathcal{A}), f \in C_{c}(G, \mathcal{A})\right\}$ of operators such that

is a *-subalgebra of $\mathcal{B}\left(L_{2}(G, \mathcal{A})\right)$.

$$
\Lambda_{f}(g)=f * g, \forall g \in L_{2}(G, \mathcal{A})
$$

\section{Proof.}

(i) For all $f \in C_{c}(G, \mathcal{A}), g \in L_{2}(G, \mathcal{A})$, we have:

$$
\left(\int_{G}\|(f * g)(x)\|_{\mathcal{A}}^{2} d x\right)^{1 / 2}=\left(\int_{G}\left\|\int_{G} f(y) g\left(y^{-1} x\right) d y\right\|_{\mathcal{A}}^{2} d x\right)^{1 / 2}
$$




$$
\begin{aligned}
& \leq\left(\int_{G}\left(\int_{G}\left\|f(y) g\left(y^{-1} x\right)\right\|_{\mathcal{A}} d y\right)^{2} d x\right)^{1 / 2} \\
& \leq \int_{G}\|f(y)\|_{\mathcal{A}}\left(\int_{G}\left\|g\left(y^{-1} x\right)\right\|_{\mathcal{A}}^{2} d x\right)^{1 / 2} d y \quad \text { (by Minkowski) } \\
& =\int_{G}\|f(y)\|_{\mathcal{A}}\left(\int_{G}\|g(x)\|_{\mathcal{A}}^{2} d x\right)^{1 / 2} d y \\
& \leq\|f\|_{\infty}\|g\|_{2}
\end{aligned}
$$

Thus, $f * g \in L_{2}(G, \mathcal{A})$ and $\exists C>0,\|f * g\|_{2} \leq C\|g\|_{2}$.

(ii) From (i), it is clear that for each $f \in C_{c}(G, \mathcal{A}), \Lambda_{f} \in \mathcal{B}\left(L_{2}(G, \mathcal{A})\right.$ ).

-Step 1: $C_{c}(G, \mathcal{A})$ is a *-algebra

It is easy to check that, $C_{c}(G, \mathcal{A})$ endowed with the convolution product is an algebra. Set $*_{\mathcal{A}}$ the involution in $\mathcal{A}$, then the mapping ${ }^{\sim}: f \mapsto \tilde{f}$ such that $\tilde{f}(s)=\left(f\left(s^{-1}\right)\right)^{* \mathcal{A}}$ is an involution of $C_{c}(G, \mathcal{A})$. In fact, $\forall \lambda \in \mathbb{C}, \forall f, g \in$ $C_{C}(G, \mathcal{A}), \forall x \in G$,

$$
\begin{aligned}
\widetilde{f * g}(x) & =\left((f * g)\left(x^{-1}\right)\right)^{* \mathcal{A}} \\
& =\left(\int_{G} f(y) g\left(y^{-1} x^{-1}\right) d y\right) \\
& =\int_{G}\left(f(y) g\left(y^{-1} x^{-1}\right)\right)^{*_{\mathcal{A}}} d y \\
& =\int_{G}\left(g\left(y^{-1} x^{-1}\right)\right)^{*_{\mathcal{A}}}(f(y))^{*_{\mathcal{A}}} d y \\
& =\int_{G}(\tilde{g}(x y))\left(\tilde{f}\left(y^{-1}\right)\right) d y \\
& =\int_{G}(\tilde{g}(z))\left(\tilde{f}\left(z^{-1} x\right)\right) d z \\
& =\tilde{g} * \tilde{f}(x) \\
& \Rightarrow(\widetilde{f * g})=\tilde{g} * \tilde{f} .
\end{aligned}
$$

Trivially,

$$
(\lambda \widetilde{f+g})=\bar{\lambda} \tilde{f}+\tilde{g}, \quad \widetilde{(\tilde{f}})=f .
$$

-Step 2: The space $\mathcal{B}\left(L_{2}(G, \mathcal{A})\right)$ is a *-algebra

Like $C_{c}(G, \mathcal{A})$, the space $L_{2}(G, \mathcal{A})$ is a $*$-algebra under the convolution product and the involution denoted by ${ }^{\sim}$. Moreover $\mathcal{B}\left(L_{2}(G, \mathcal{A})\right)$ is a -algebra if endowed with:

-the inner product $T_{1} \circ T_{2}: f \mapsto T_{1}\left(T_{2} f\right)$,

-and the involution $\quad{ }^{\star}: T \mapsto T^{\star}$ such that $\left\langle T^{\star} g, h\right\rangle_{L_{2}}=\langle g, T h\rangle_{L_{2}}$, for all $g, h \in L_{2}(G, \mathcal{A})$.

-Step 3: The space $\mathcal{T}(G, \mathcal{A})$ is a *-subalgebra of $\mathcal{B}\left(L_{2}(G, \mathcal{A})\right)$ 


$$
\begin{aligned}
&\left(\lambda \Lambda_{f_{1}}+\Lambda_{f_{2}}\right)(g)=\lambda f_{1} * g+f_{2} * g \\
&=\left(\lambda f_{1}+f_{2}\right) * g \\
&= \Lambda_{\lambda f_{1}+f_{2}}(g) \\
& \Rightarrow \lambda \Lambda_{f_{1}}+\Lambda_{f_{2}}= \Lambda_{\lambda f_{1}+f_{2}} \in \mathcal{T}(G, \mathcal{A}) . \\
&\left\langle\left(\Lambda_{f}\right)^{\star}(g), h\right\rangle_{L_{2}(G, \mathcal{A})}=\left\langle g, \Lambda_{f} h\right\rangle_{L_{2}(G, \mathcal{A})} \\
&=\langle g, f * h\rangle_{L_{2}(G, \mathcal{A})} \\
&=\langle\tilde{f} * g, h\rangle_{L_{2}(G, \mathcal{A})} \\
&=\left\langle\Lambda_{\tilde{f}}(g), h\right\rangle_{L_{2}(G, \mathcal{A})} \\
&\left\langle\left(\Lambda_{f}\right)^{\star}(g), h\right\rangle_{L_{2}(G, \mathcal{A})}=\left\langle\Lambda_{\tilde{f}}(g), h\right\rangle_{L_{2}(G, \mathcal{A})} \Rightarrow\left(\Lambda_{f}\right)^{\star}=\Lambda_{\tilde{f}} \in \mathcal{T}(G, \mathcal{A}) . \\
& \Rightarrow\left(\lambda \Lambda_{f_{1}}+\Lambda_{f_{2}}\right)^{\star}= \\
& \\
&\left(\lambda \Lambda_{f_{1}}+\Lambda_{f_{2}}\right)^{\star}=\Lambda_{\left(\lambda \widetilde{f_{1}+f_{2}}\right)} \\
&=\Lambda_{\bar{\lambda} \widetilde{f_{1}}+\widetilde{f_{2}}} \\
&\left.=\bar{\lambda} \Lambda_{\widetilde{f_{1}}}+\Lambda_{\widetilde{f_{2}}}\right)^{\star}+\left(\Lambda_{f_{2}}\right)^{\star} .
\end{aligned}
$$

Since,

$$
\begin{aligned}
\left(\Lambda_{f_{1}} \circ \Lambda_{f_{2}}\right) g & =\Lambda_{f_{1}}\left(\Lambda_{f_{2}} g\right) \\
& =f_{1} *\left(f_{2} * g\right) \\
& =\left(f_{1} * f_{2}\right) * g \\
& =\Lambda_{f_{1} * f_{2}} g \\
\Rightarrow \Lambda_{f_{1}} \circ \Lambda_{f_{2}} & =\Lambda_{f_{1} * f_{2}},
\end{aligned}
$$

then,

$$
\begin{gathered}
\left(\Lambda_{f_{1}} \circ \Lambda_{f_{2}}\right)^{*}=\Lambda_{\left(\widetilde{f_{1} * f_{2}}\right)} \\
=\Lambda_{\widetilde{f_{2}} * \widetilde{f_{1}}} \\
=\Lambda_{\widetilde{f_{2}}} \circ \Lambda_{\widetilde{f_{1}}}
\end{gathered}
$$

The rest of the proof is obvious.

Remark 3.2 The previous proposition is always true, if $C_{c}(G, \mathcal{A})$ is replaced by $L_{1}(G, \mathcal{A})$. 
Corollary 3.3 If $\mathcal{A}$ is an $H^{*}$-algebra endowed with its natural operator space structure, then $\forall f \in L_{1}(G, \mathcal{A}), \Lambda_{f}$ is completely bounded. More precisely, $\mathcal{T}(G, A) \subset c b\left(L_{1}(G, \mathcal{A})\right)$.

Proof. For $n \in \mathbb{N}^{*}$, consider the mapping

$$
\begin{gathered}
\Lambda_{f}^{(n)}: M_{n}\left(L_{2}(G, \mathcal{A})\right) \rightarrow M_{n}\left(L_{2}(G, \mathcal{A})\right) \\
\left(g_{i j}\right)_{1 \leq i, j \leq n} \mapsto\left(\Lambda_{f} g_{i j}\right)_{1 \leq i, j \leq n} \\
\left\|\Lambda_{f}^{(n)}\right\|=\sup \left\{\left\|\Lambda_{f}^{(n)}\left(\left(g_{i j}\right)_{1 \leq i, j \leq n}\right)\right\|_{M_{n}\left(L_{2}(G, \mathcal{A})\right)}:\left\|\left(g_{i j}\right)_{1 \leq i, j \leq n}\right\|_{M_{n}\left(L_{2}(G \mathcal{A})\right)} \leq 1\right\} \\
=\sup \left\{\left\|\left(\Lambda_{f} g_{i j}\right)_{1 \leq i, j \leq n}\right\|_{M_{n}\left(L_{2}(G, \mathcal{A})\right)}: \sup _{1 \leq i, j \leq n}\left\|g_{i j}\right\|_{L_{2}(G, \mathcal{A})} \leq 1\right\} \\
=\quad \\
\| \sup _{1 \leq i, j \leq n}\left\{\left\|\Lambda_{f} g_{i j}\right\|_{2}:\left\|g_{i j}\right\|_{2} \leq 1\right\} \\
\quad \leq \sup _{1 \leq i, j \leq n}\left\{\left\|f * g_{i j}\right\|_{2}:\left\|g_{i j}\right\|_{2} \leq 1\right\} \\
\leq \sup _{1 \leq i, j \leq n}\left\{\|f\|_{1}\left\|g_{i j}\right\|:\left\|g_{i j}\right\|_{2} \leq 1\right\}
\end{gathered}
$$

So $\sup _{n \geq 1}\left\|\Lambda_{f}^{(n)}\right\| \leq\|f\|_{1}<\infty$,

And $\Lambda_{f}$ is completely bounded.

Definition 3.4 Assume $\mathcal{A}$ is an $H^{*}$-algebra.

For a locally compact group $G$, we denote by $C^{*}(G, \mathcal{A})$ the (vector-valued) $C^{*}$-algebra of $G$, which is $G$ the $C^{*}$-envelopping algebra of $L_{1}(G, \mathcal{A})$, i.e. the completion of $C_{c}(G, \mathcal{A})$ with respect to the largest $C^{*}$-norm

$$
\|f\|_{*_{\infty}}=\sup _{\pi}\|\pi(f)\|
$$

where $\pi$ ranges over all non-degenerates $*$-representations of $C_{c}(G, \mathcal{A})$ on Hilbert spaces.

Definition 3.5 Let $G$ be a locally compact group and $\mathcal{A}$ an $H^{*}$-algebra.

The (vector-valued) reduced group $C^{*}$-algebra $C_{r}^{*}(G, \mathcal{A})$ is the completion of $C_{c}(G, \mathcal{A})$ with respect to the norm

$$
\sup _{g \in L_{2}(G, A)}\left\{\|f * g\|_{L_{2}(G, \mathcal{A})}:\|g\|_{2} \leq 1\right\} .
$$

Proposition 3.6 The space $C_{c}(G, \mathcal{A})$ is isometrically isomorphic to the space $\mathcal{T}(G, \mathcal{A})$.

Proof. The operators $\Lambda_{f}$ determine the bijective linear map

$$
\begin{array}{ccc}
\Lambda: C_{c}(G, \mathcal{A}) & \rightarrow & \mathcal{T}(G, \mathcal{A}) \subset \mathcal{B}\left(L_{2}(G, \mathcal{A})\right) \\
f & \mapsto & \Lambda_{f} .
\end{array}
$$

Moreover, for any $f \in C_{c}(G, \mathcal{A})$, 


$$
\begin{aligned}
\|\Lambda(f)\|_{\mathcal{B}\left(L_{2}(G, \mathcal{A})\right)}= & \left\|\Lambda_{f}\right\|_{\mathcal{B}\left(L_{2}(G, \mathcal{A})\right)} \\
= & \sup _{g \in L_{2}(G, \mathcal{A})}\left\{\left\|\Lambda_{f} g\right\|_{L_{2}(G, \mathcal{A})}:\|g\|_{2} \leq 1\right\} \\
= & \sup _{g \in L_{2}(G, \mathcal{A})}\left\{\|f * g\|_{L_{2}(G, \mathcal{A})}:\|g\|_{2} \leq 1\right\} \\
& =\|f\|_{C_{r}^{*}},
\end{aligned}
$$

which completes the proof.

Corollary 3.7 Assume $\mathcal{A}$ is an $H^{*}$-algebra and $G$ a locally compact group. The norm

is a $C^{*}$-norm on $C_{r}^{*}(G, \mathcal{A})$.

$$
\|f\|_{C_{r}^{*}:=} \sup _{g \in L_{2}(G, \mathcal{A})}\left\{\|f * g\|_{2}:\|g\|_{2} \leq 1\right\}
$$

Proof. We already know that $C_{c}(G, \mathcal{A})$ is a $*$-algebra, and $\left(C_{r}^{*}(G, \mathcal{A}),\|\cdot\|_{C_{r}^{*}}\right)$ is a Banach space. Moreover, using Proposition 3.6 we have :

(i) -Submultiplicative property:

$$
\begin{aligned}
\left\|f_{1} * f_{2}\right\|_{C_{r}^{*}} & =\quad\left\|\Lambda_{f_{1}} \circ \Lambda_{f_{2}}\right\|_{\mathcal{B}\left(L_{2}(G, \mathcal{A})\right)} \\
& \leq\left\|\Lambda_{f_{1}}\right\|_{\mathcal{B}\left(L_{2}(G, \mathcal{A})\right)}\left\|\Lambda_{f_{2}}\right\|_{\mathcal{B}\left(L_{2}(G, \mathcal{A})\right)}=\left\|f_{1}\right\|_{C_{r}^{*}}\left\|f_{2}\right\|_{C_{r}^{*}}
\end{aligned}
$$

(ii) $\quad-\|\cdot\|_{C_{r}^{*}}$ is a normed algebra:

$$
\|\tilde{f}\|_{C_{r}^{*}}=\left\|\left(\Lambda_{f}\right)^{\star}\right\|_{\mathcal{B}\left(L_{2}(G, \mathcal{A})\right)}=\left\|\Lambda_{f}\right\|_{\mathcal{B}\left(L_{2}(G, \mathcal{A})\right)}=\|f\|_{C_{r}^{*}}
$$

(iii) -The $C^{*}$-property:

$$
\|\tilde{f} * f\|_{C_{r}^{*}}=\left\|\left(\Lambda_{f}\right)^{\star} \circ \Lambda_{f}\right\|=\left\|\Lambda_{f}\right\|^{2}=\|f\|^{2} .
$$

Following Proposition 3.6, the reduced group $C^{*}$-algebra $C_{r}^{*}(G, \mathcal{A})$ can be defined equivalently as follows:

\section{Definition 3.8 (Definition 3.5 bis)}

Let $G$ be a locally compact group and $\mathcal{A}$ an $H^{*}$-algebra. The (vector-valued) reduced group $C^{*}$-algebra $C_{r}^{*}(G, \mathcal{A})$ is the closure of the space $\mathcal{T}(G, \mathcal{A})$, with respect to the operator norm on $\mathcal{B}\left(L_{2}(G, \mathcal{A})\right)$.

Remark 3.9 In the second definition, $C_{r}^{*}(G, \mathcal{A})$ is indeed a $C^{*}$-algebra. In fact, $C_{r}^{*}(G, \mathcal{A})$ is a closed self-adjoint subalgebra of the $C^{*}$-algebra $\mathcal{B}\left(L_{2}(G, \mathcal{A})\right)$ with respect to the $C^{*}$-norm of $\mathcal{B}\left(L_{2}(G, \mathcal{A})\right)$ (the operator norm). By following this definition, one can conclude that $C_{r}^{*}(G, \mathcal{A})$ is the $C^{*}$-algebra generated by the image of the left regular representation of $C_{c}(G, \mathcal{A})$ on $L_{2}(G, \mathcal{A})$. 


\section{The generalized group von Neumann algebra $\operatorname{VN}(G, \mathcal{A})$}

Definition 4.1 The (vector-valued) group von Neumann algebra $\operatorname{VN}(G, \mathcal{A})$ of $G$ is the enveloping von Neumann algebra of $C^{*}(G, \mathcal{A})$, i.e. the weak operator topology closure of $C_{r}^{*}(G, \mathcal{A})$.

Remark 4.2 -Considering the previous assertions in Remark 3.9 about the reduced $C^{*}$-algebra, one can also define the (vector-valued) group von Neumann algebra as follows:

$$
V N(G, \mathcal{A})=\{\lambda(s): s \in G\}
$$

where $\lambda: G \rightarrow \mathcal{B}\left(L_{2}(G, \mathcal{A})\right)$ is the left regular representation of $G$.

$$
\begin{array}{clll}
\lambda(s): L_{2}(G, \mathcal{A}) & \rightarrow & & L_{2}(G, \mathcal{A}) \\
f & \mapsto & g, & g(t)=f\left(s^{-1} t\right)
\end{array}
$$

-Naturally, $A(G, \mathbb{C})=A(G)$ and $V N(G, \mathbb{C})=V N(G)$.

Proposition 4.3 The Fourier algebra $A(G, \mathcal{A})$ is isometrically isomorphic to the predual of the group von Neumann algebra $\operatorname{VN}(G, \mathcal{A})$.

Proof. Consider the mapping

$$
\begin{array}{ccc}
\phi: V N(G, \mathcal{A}) & \rightarrow & (A(G, \mathcal{A}))^{*} \\
v & \mapsto & \phi(v)
\end{array}
$$

such that if $u=f * \tilde{g} \in A(G, \mathcal{A})$, then $\phi(v)(u)=\left\langle\Lambda_{v} f, g\right\rangle=\int_{G}\left\langle\left(\Lambda_{v} f\right)(x), g(x)\right\rangle_{\mathcal{A}} \mathrm{d} \mu(\mathrm{x})$.

We know that $C_{r}^{*}(G, \mathcal{A})$ is a $C^{*}$-subalgebra of $\mathcal{B}\left(L_{2}(G, \mathcal{A})\right)$ with strong closure $V N(G, \mathcal{A})$, so the closed unit ball of $C_{r}^{*}(G, \mathcal{A})$ is strongly dense in the unit ball of $V N(G, \mathcal{A})$ (Kaplansky theorem of density). Thus, there exists a sequence $\left(w_{n}\right)$ in $C_{c}(G, \mathcal{A})$ such that $\left\|w_{n}\right\|_{C_{r}^{*}} \leq\|v\|_{C_{r}^{*}}$ and $\left(\Lambda_{w_{n}}\right) \underset{\text { strongly }}{\longrightarrow} \Lambda_{v}$. Moreover,

$$
\begin{aligned}
|\phi(v)(u)| & =\lim _{n}\left|\left\langle\Lambda_{w_{n}} f, g\right\rangle\right| \\
& =\lim _{n}\left|\int_{G}\left\langle\Lambda_{w_{n}} f(x), g(x)\right\rangle_{\mathcal{A}} d \mu(x)\right| \\
& =\lim _{n}\left|\int_{G}\left\langle w_{n} * f(x), g(x)\right\rangle_{\mathcal{A}} d \mu(x)\right| \\
& =\lim _{n} \mid \int_{G}\left\langle\int_{G} w_{n}(y) f\left(y^{-1} x\right) d y,\left.g(x)\right|_{\mathcal{A}} d \mu(x)\right| \\
& =\lim _{n}\left|\int_{G}\right| w_{n}(y),\left.\int_{G} g(x)\left(f\left(y^{-1} x\right)\right)^{*} \mathcal{A} d x\right|_{\mathcal{A}} d y \mid \\
& =\lim _{n} \mid \int_{G}\left\langle w_{n}(y),\left.\int_{G} g(y z)(f(z))^{* \mathcal{A}} d z\right|_{\mathcal{A}} d y\right|
\end{aligned}
$$




$$
\begin{aligned}
& =\lim _{n}\left|\int_{G}\right| w_{n}(y),\left.\left(\int_{G} f(z)(g(y z))^{* A} d z\right)^{*_{\mathcal{A}}}\right|_{\mathcal{A}} d y \mid \\
& =\lim _{n}\left|\int_{G}\right| w_{n}(y),\left.\left(\int_{G} f(z) g\left(z^{-1} y^{-1}\right) d z\right)^{*_{\mathcal{A}}}\right|_{\mathcal{A}} d y \mid \\
& =\quad \lim _{n}\left|\int_{G}\left\langle w_{n}(y),\left(f * g\left(y^{-1}\right)\right)^{* \mathcal{A}}\right\rangle_{\mathcal{A}} d y\right| \\
& =\lim _{n}\left|\int_{G}\left\langle w_{n}(y), \forall t(y)\right\rangle_{\mathcal{A}} d y\right| \\
& \leq \quad \lim _{n}\left\|w_{n}\right\|_{C_{r}^{*}}\|u\|_{A(G, \mathcal{A})} \\
& \leq \quad\|v\|_{C_{r}^{*}}\|u\|_{A(G, \mathcal{A})} \\
& \Rightarrow \quad\|\phi(v)\| \leq\|v\|
\end{aligned}
$$

Moreover, we have

$$
\begin{aligned}
\|v\|_{C_{r}^{*}} & =\sup _{f \in L_{2}(G, \mathcal{A})}\left\{\|h * f\|_{2}:\|f\|_{2} \leq 1\right\} \\
& =\sup _{f, g \in L_{2}(G, \mathcal{A})}\left\{\left|\langle h * f, g\rangle_{\mathrm{L}_{2}}\right|:\|f\|_{2} \leq 1,\|f\|_{2} \leq 1\right\} \\
& \geq \sup _{f, g \in L_{2}(G, \mathcal{A})}\{|\phi(v)(u)|:\|u\| \leq 1\} \\
& \geq
\end{aligned}
$$

The linearity of $\phi$ is obvious, let us prove the injectivity. Assume $\phi(T)=0$, then for all $f, g \in L_{2}(G, \mathcal{A})$,

$$
\begin{aligned}
\phi(T)(f * \tilde{g})=0 & \Rightarrow & \int_{G}\left\langle T(y),(f * g(y))^{* \mathcal{A}}\right\rangle_{\mathcal{A}} d y=0 \\
& \Rightarrow & T(y)=0 \forall y \in G \\
& \Rightarrow & T=0
\end{aligned}
$$

Conversely, assume $\varphi \in(A(G, \mathcal{A}))^{*}$ and let $f, g \in L_{2}(G, \mathcal{A})$, then

$$
\begin{aligned}
|\varphi(f * g)| & \leq\|\varphi\|_{(A(G, \mathcal{A}))^{*}\|f * g\|_{A(G, \mathcal{A})} \quad \text { (since } \varphi \text { is continuous) }} \\
& \leq \varphi \varphi\|f\|_{2}\|g\|_{2} \\
& \Rightarrow \sup _{f, g \in L_{2}(G \mathcal{A})}\left\{|\varphi(f * g)|:\|f\|_{2} \leq 1,\|g\|_{2} \leq 1\right\} \leq\|\varphi\|_{(A(G, \mathcal{A}))^{*}}
\end{aligned}
$$

Then, there exists a linear map $\mathcal{V}_{\varphi} \in \mathcal{B}\left(L_{2}(G, \mathcal{A})\right)$ such that $\left\langle\mathcal{V}_{\varphi} f, g\right\rangle=\varphi(f * g)$ and $\left\|\mathcal{v}_{\varphi}\right\| \leq\|\varphi\|$.

Let us prove that $\mathcal{V}_{\varphi}$ commutes with convolution:

$\forall f, g \in L_{2}(G, \mathcal{A}), \forall h \in C_{c}(G, \mathcal{A})$, we have

$$
\begin{array}{rlc}
\left\langle\mathcal{V}_{\varphi}(f * h), g\right\rangle & = & \varphi((f * h) * \tilde{g})=\varphi(f *(h * \tilde{g})) \\
& = & \varphi(f *(\widetilde{g * \tilde{h}})) \\
& = & \left\langle\mathcal{V}_{\varphi} f, g * \tilde{h}\right\rangle \\
& =\left\langle\left(\mathcal{V}_{\varphi} f\right) * h, g\right\rangle,
\end{array}
$$


which implies that $\mathcal{V}_{\varphi}(f * h)=\left(\mathcal{V}_{\varphi} f\right) * h$, and $\mathcal{V}_{\varphi}$ is an element of $V N(G, \mathcal{A})$.

Let $C^{b}(G)$ be the space of all bounded continuous functions from $G$ to $\mathbb{C}$, a function $f \in C^{b}(G)$ such that $\forall g \in$ $A(G), f g \in A(G)$ is said to be a multiplier of $A(G)$. The space of all completely bounded multipliers on $A(G)$ is denoted by $M_{c b}(A(G))$. In a similar way, we define the space of completely bounded multipliers on $A(G, \mathcal{A})$ and the space of completely bounded vector-valued multipliers on $A(G, \mathcal{A})$.

Definition 4.4 Let $G$ be a locally compact group, $\mathcal{A}$ an $H^{*}$-algebra and $C^{b}(G, \mathcal{A})$ the space of all bounded continuous functions from $G$ to $\mathcal{A}$. Let $V_{1} \subset C^{b}(G, \mathcal{A})$ and $V_{2} \subset A(G, \mathcal{A})$ two vector spaces. We denote by $M_{c b} A(G, \mathcal{A}) \subset C^{b}(G, \mathcal{A})\left(\right.$ resp. $\left.M_{c b} V_{2}\right)$ the space of completely bounded multipliers on $A(G, \mathcal{A})$ (resp. on $V_{2}$ ), i.e. the collection of functions $f \in C^{b}(G, \mathcal{A})$ (resp. on $V_{1}$ ) such that $f g \in A(G, \mathcal{A})$ (resp. fg $\in V_{2}$ ) for each $g \in A(G, \mathcal{A})$ (resp. for each $g \in V_{2}$ ) and the operator

$$
\begin{array}{cl}
M_{f}: A(G, \mathcal{A}) & \rightarrow A(G, \mathcal{A})\left(\text { resp. } V_{2} \rightarrow V_{2}\right) \\
g & \mapsto f g,
\end{array}
$$

is completely bounded,

where

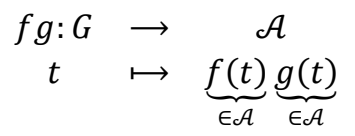

Remark 4.5 We denote by $\operatorname{MA}(G, \mathcal{A})$ the space of all multipliers of $A(G, \mathcal{A})$. Let $\lambda$ be the left regular representation of $C_{c}(G, \mathcal{A})$ on $L_{2}(G, \mathcal{A})$. As in the case of multipliers of $A(G)$ (cf [9], Introduction), each $f \in$ $\operatorname{MA}(G, \mathcal{A})$ generates an operator $M_{f}$ on $A(G, \mathcal{A})$ whose transpose defines a $\sigma$-weakly continuous operator $M_{f}$ on $V N(G, \mathcal{A})$ such that $M_{f} \lambda(s)=f(s) \lambda(s)$, for $s \in V N(G, \mathcal{A})$,

Definition 4.6 We define $A^{0}(G, \mathcal{A})$ as the following vector space.

$$
A^{0}(G, \mathcal{A})=\left\{\sum_{j=1}^{n} a_{j} g_{j}: a_{j} \in \mathcal{A}, g_{j} \in A(G), n \in \mathbb{N}^{*}\right\} .
$$

We also define the vector space $C^{b_{0}}(G, \mathcal{A})$ as follows.

$$
C^{b_{0}}(G, \mathcal{A})=\left\{\sum_{j=1}^{n} a_{j} g_{j}: a_{j} \in \mathcal{A}, g_{j} \in C^{b}(G), n \in \mathbb{N}^{*}\right\} .
$$

Theorem 4.7 Let $G$ be a locally compact group and let $\mathcal{A}$ be a unital and commutative $H^{*}$-algebra. The following assertions hold:

(i) $\quad A^{0}(G, \mathcal{A}) \subset A(G, \mathcal{A}), A^{0}(G, \mathcal{A})$ is dense in $A(G, \mathcal{A})$.

(ii) $\quad C^{b_{0}}(G, \mathcal{A}) \subset C^{b}(G, \mathcal{A}), C^{b_{0}}(G, \mathcal{A})$ is dense in $C^{b}(G, \mathcal{A})$.

\section{Proof.}

(i) Let $a \in \mathcal{A}$ and $f \in A(G)$. There exists $f_{1}, f_{2} \in L_{2}(G)$ such that $f=f_{1} * f_{2}$. Consider the function

$$
\begin{array}{ccc}
a f: G & \rightarrow & L_{2}(G, \mathcal{A}) \\
t & \mapsto & a(f(t)) .
\end{array}
$$


We have, $a f=a f_{1} * \widetilde{f_{2}}=\left(a f_{1}\right) *\left(1_{\mathcal{A}} \widetilde{f_{2}}\right)=\underbrace{\left(a f_{1}\right)}_{\in L_{2}(G, \mathcal{A})} *(\underbrace{\widetilde{\mathcal{A}_{2} f_{2}}}_{\in L_{2}(G, \mathcal{A})})$ which implies that $a f \in A(G, \mathcal{A})$, and finally $A^{0}(G, \mathcal{A}) \subset A(G, \mathcal{A})$

Let $f=g * h \in A(G, \mathcal{A})$, with $g, h \in L_{2}(G, \mathcal{A})$

Set

$$
L_{2}^{0}(G, \mathcal{A})=\left\{\sum_{j=1}^{n} a_{j} g_{j}: a_{j} \in \mathcal{A}, g_{j} \in L_{2}(G), n \in \mathbb{N}^{*}\right\},
$$

, It is know that $L_{2}^{0}(G, \mathcal{A})$ is dense in $L_{2}(G, \mathcal{A})$, then for all $\varepsilon>0$, there exist $g_{\varepsilon}, h_{\varepsilon} \in L_{2}^{0}(G, \mathcal{A})$ such that

$$
\left\|g-g_{\varepsilon}\right\|_{2} \leq \frac{\varepsilon}{2\left(1+M_{\varepsilon}\right)} \text { and }\left\|h-h_{\varepsilon}\right\|_{2} \leq \frac{\varepsilon}{2\left(1+M_{\varepsilon}\right)},
$$

Where $M_{\varepsilon}=\sup \left\{\left\|g_{\varepsilon}\right\|_{2} ;\left\|h_{\varepsilon}\right\|_{2}\right\}$.

Moreover, there exist $n, m \in \mathbb{N}^{*}, a_{\varepsilon, 1}, a_{\varepsilon, 2}, \ldots, a_{\varepsilon, n}, b_{\varepsilon, 1}, b_{\varepsilon, 2}, \ldots, b_{\varepsilon, m} \in \mathcal{A}$ and $g_{\varepsilon, 1}, g_{\varepsilon, 2}, \ldots, g_{\varepsilon, n}, h_{\varepsilon, 1}, h_{\varepsilon, 2} \ldots, h_{\varepsilon, m} \in L_{2}(G, \mathcal{A})$ such that

$$
\begin{aligned}
g_{\varepsilon} * \tilde{h}_{\varepsilon} & =\left(\sum_{i=1}^{n} a_{\varepsilon, i} g_{\varepsilon, i}\right) *\left(\sum_{i=1}^{m} b_{\varepsilon, j} \tilde{h}_{\varepsilon, j}\right) \\
& =\sum_{\mathrm{i}=1}^{\mathrm{n}} \sum_{\mathrm{j}=1}^{\mathrm{m}} a_{\varepsilon, i} b_{\varepsilon, j} \underbrace{\left(g_{\varepsilon, i} * \tilde{h}_{\varepsilon, j}\right)}_{\in \mathrm{A}(\mathrm{G})}
\end{aligned}
$$

which means that $g_{\varepsilon} * \tilde{h}_{\varepsilon}$ is an element of $A^{0}(G, \mathcal{A})$.

Set $f_{\varepsilon}=g_{\varepsilon} * \tilde{h}_{\varepsilon}$, we have,

$$
\begin{array}{rlr}
\left\|f-f_{\varepsilon}\right\|_{A(G, \mathcal{A})} & = & \left\|g * \tilde{h}-g_{\varepsilon} * \tilde{h}_{\varepsilon}\right\|_{A(G \mathcal{A})} \\
& \leq & \left\|g_{\varepsilon} *\left(\tilde{h}-\tilde{h}_{\varepsilon}\right)\right\|_{A(G, \mathcal{A})}+\left\|\left(g-g_{\varepsilon}\right) * \tilde{h}\right\|_{A(G, \mathcal{A})} \\
& \leq & \left\|g_{\varepsilon}\right\|_{2}\left\|h-h_{\varepsilon}\right\|_{2}\left\|g-g_{\varepsilon}\right\|_{2}\|h\|_{2} \\
& \leq & \varepsilon\left(\frac{M_{\varepsilon}}{1+M_{\varepsilon}}\right) \\
& \leq & \varepsilon
\end{array}
$$

Hence, $A^{0}(G, \mathcal{A})$ is dense in $A(G, \mathcal{A})$.

(ii) This follows by using the same method as in (i).

Remark 4.8 If $\mathcal{A}=\mathbb{C}$, then $A^{0}(G, \mathbb{C})=A(G)$ and $C^{b_{0}}(G, \mathbb{C})=C^{b}(G)$.

Corollary 4.9 Let $G$ be a locally compact group and let $\mathcal{A}$ be a unital commutative $H^{*}$-algebra. $A(G) \otimes \mathcal{A}$ is isometrically isomorphic to a dense subspace of $A(G, \mathcal{A})$. 
Proof. It is obvious that the space $A^{0}(G, \mathcal{A})$ is isometrically isomorphic to $A(G) \otimes \mathcal{A}$, so using the previous theorem, we are done.

Proposition 4.10 Let $\left(\xi_{j}\right)_{j \in J}$ be an orthonormal basis of an $H^{*}$-algebra $\mathcal{A}$. For each $g \in A^{0}(G, \mathcal{A})$, there exists a family of functions in $A(G)$ such that

Proof. Let $g \in A^{0}(G, \mathcal{A})$, then

$$
g(t)=\sum_{j \in J} g_{j}(t) \xi_{j}
$$

$$
g=\sum_{i=1}^{n} a_{i} h_{i},
$$

with $a_{i} \in \mathcal{A}$ and $h_{i} \in A(G)$. Since $\mathcal{A}$ has a Hilbert space structure with $\left(\xi_{j}\right)_{j \in J}$ as an orthonormal basis, then for all $t \in G$ we have:

$$
\begin{aligned}
g(t) & = \\
& =\sum_{i=1}^{n}\left(\sum_{j \in J} \lambda_{i}^{j} \xi_{i}\left(h_{i}(t)\right)\left(h_{i}(t)\right) \text { where } \lambda_{i}^{j} \in \mathrm{C}\right. \\
& =\quad \sum_{j \in J}\left(\sum_{i=1}^{n} \lambda_{i}^{j}\left(h_{i}(t)\right)\right) \xi_{j} \\
& =\sum_{j \in J} g_{j}(t) \xi_{j} \text { with } g_{j}=\sum_{i=1}^{n} \lambda_{i}^{j} h_{i} \in A(G) .
\end{aligned}
$$

Lemma 4.11 Let $G$ be a locally compact group and let $\mathcal{A}$ be $a$ unital and commutative $H^{*}$-algebra. A function $f=\sum_{i=1}^{n} f_{i} \in C^{b_{0}}(G, \mathcal{A})$ is a completely bounded multiplier on $A^{0}(G, \mathcal{A})$ if and only if for each $1 \leq i \leq n, f_{i}$ is a completely bounded multiplier on $A(G)$.

Proof. Assume $f$ is a completely bounded multiplier on $A^{0}(G, \mathcal{A})$, then for all $g \in A^{0}(G, \mathcal{A}), f g \in A^{0}(G, \mathcal{A})$,

$$
\begin{aligned}
& \text { i.e. } \sum_{i=1}^{n} a_{i} f_{i} \sum_{j=1}^{m} b_{j} g_{j}=\sum_{i=1}^{n} \sum_{j=1}^{m} a_{i} b_{j} f_{i} g_{j} \in A^{0}(G, \mathcal{A}) \\
& \text { i.e. for all } 1 \leq i \leq n, 1 \leq j \leq m, \quad f_{i} g_{j} \in A(G) .
\end{aligned}
$$

Since for all $b \in \mathcal{A}$ and for all $h \in A(G)$ we have $b h \in A^{0}(G, \mathcal{A})$

then by setting $m=1, b_{1}=b$ and $g_{1}=h$, (1) becomes

$\forall h \in A(G)$, and $1 \leq i \leq n, f_{i} h \in A(G)$.

Moreover, since the operator,

$$
\begin{array}{ccc}
\mathcal{M}_{f}: A^{0}(G, \mathcal{A}) & \rightarrow & A^{0}(G, \mathcal{A}) \\
g & \mapsto & f g
\end{array}
$$

is completely bounded, it is obvious that for each $1 \leq i \leq n$, the operator 


$$
\begin{array}{ccc}
m_{f_{i}}: A(G) & \rightarrow & A(G) \\
h & \mapsto & f_{i} h
\end{array}
$$

is also completely bounded. In fact, since $\mathcal{M}_{f}$ is $c . b$. on $A^{0}(G, \mathcal{A})$, we have

$$
\sup _{k \in \mathbb{N}^{*}}\left\|I_{M_{k}} \otimes \mathcal{M}_{f}\right\|_{\mathcal{B}\left(M_{k} \otimes A^{0}(G, \mathcal{A})\right)}<\infty .
$$

So $\forall \alpha \in M_{k}, \forall h \in A(G), \forall b \in \mathcal{A}$ such that $\|\alpha \otimes b h\| \leq 1$, we have

with $1 \leq i \leq n$. This implies that

$$
\sup _{k \in \mathbb{N}^{*}}\left\|\alpha \otimes\left(\left(a_{i} f_{i}\right)(b h)\right)\right\|_{M_{k} \otimes A^{0}(G, \mathcal{A})}<\infty
$$

$\left\|a_{i}\right\|_{\mathcal{A}} \sup _{k \in \mathbb{N}^{*}}\left\|\left(I_{M_{k}} \otimes m_{f_{i}}\right)(\alpha \otimes h)\right\|_{M_{k} \otimes A(G)}<\infty, \forall \alpha \in M_{k}, \forall h \in A(G)$ such that $\|\alpha \otimes h\| \leq 1$.

Hence $\sup _{k \in \mathbb{N}^{*}}\left\|I_{M_{k}} \otimes m_{f_{i}}\right\|_{B\left(M_{k} \otimes A(G)\right)}<\infty$ and $m_{f_{i}}$ is completely bounded on $A(G)$.

We conclude that for all $1 \leq i \leq n, f_{i}$ is a completely bounded multiplier on $A(G)$.

Conversely, if for all $1 \leq i \leq n, f_{i}$ is a completely bounded multiplier on $A(G)$, then for all $h \in A(G), f_{i} h \in A(G)$. Let $g \in A^{0}(G, \mathcal{A})$, there exists a family of elements $b_{1}, b_{2}, \cdots, b_{m} \in \mathcal{A}, g_{1}, g_{2}, \cdots, g_{m} \in A(G)\left(m \in \mathbb{N}^{*}\right)$ such that

$$
g=\sum_{j=1}^{m} b_{j} g_{j} .
$$

Thus, $f_{i} g_{j} \in A(G)$ and $a_{i} b_{j} \in \mathcal{A}$, which means that $f g \in A^{0}(G, \mathcal{A})$.

Now, let $k \in \mathbb{N}^{*}$ and $\alpha_{k} \in M_{k}$ such that $\left\|\alpha_{k} \otimes g\right\| \leq 1$, then $\left\|b_{j}\right\| \leq 1$ and $\left\|g_{j}\right\| \leq 1$ (for all $1 \leq j \leq m$ ). Set

and

$$
\omega=\sup _{1 \leq i \leq n}\left\{\left\|a_{i}\right\|_{\mathcal{A}}\left\|m_{f_{i}}\right\|_{c b(A(G))}\right\}
$$

we have:

$$
\mathcal{S}=\sup _{k \in \mathbb{N}^{*}}\left\{\left\|\left(I_{M_{k}} \otimes \mathcal{M}_{f}\right)\left(\alpha_{k} \otimes g\right)\right\|_{M_{k} \otimes_{\min } A^{0}(G, \mathcal{A})}\right\},
$$

$$
\begin{aligned}
\mathcal{S} & =\sup _{k \in \mathbb{N}^{*}}\left\{\left\|\alpha_{k} \otimes(f g)\right\|_{M_{k} \otimes_{\min } A^{0}(G, \mathcal{A})}\right\} \\
& \leq \sup _{k \in \mathbb{N}^{*}}\left\{\sum_{i=1}^{n} \sum_{j=1}^{m}\left\|\alpha_{k} \otimes a_{i} b_{j} f_{i} g_{j}\right\|_{M_{k} \otimes_{\min } A^{0}(G, \mathcal{A})}\right\} \\
& \leq \sup _{k \in \mathbb{N}^{*}}\left\{\sum_{i=1}^{n} \sum_{j=1}^{m}\left\|a_{i} b_{j}\right\|_{\mathcal{A}}\left\|\alpha_{k} \otimes\left(f_{i} g_{j}\right)\right\|_{M_{k} \otimes_{\min } \mathrm{A}(G)}\right\} \\
& \leq \sum_{i=1}^{n} \sum_{j=1}^{m}\left\|a_{i}\right\|_{\mathcal{A}}\left\|b_{j}\right\|_{\mathcal{A}_{k \in \mathbb{N}^{*}}} \sup _{k}\left\{\left(I_{M_{k}} \otimes m_{f_{i}}\right)\left(\alpha_{k} \otimes\left(g_{j}\right) \|_{M_{k} \otimes_{\min } \mathrm{A}(G)}\right\}\right. \\
& \leq \sum_{i=1}^{n}\left\|a_{i}\right\|_{\mathcal{A}} \sup _{k \in \mathbb{N}^{*}}\left\{\left\|I_{M_{k}} \otimes m_{f_{i}}\right\|_{\mathcal{B}\left(M_{k} \otimes \mathrm{A}(G)\right)}\right\} \\
& \leq \sum_{i=1}^{n}\left\|a_{i}\right\|\left\|m_{f_{i}}\right\|_{c b(A(G))}
\end{aligned}
$$




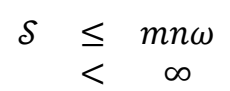

which implies that $\sup _{k \in \mathbb{N}^{*}}\left\{\left\|\left(I_{M_{k}} \otimes \mathcal{M}_{f}\right)\right\|_{\mathcal{B}\left(M_{k} \otimes A^{0}(G, \mathcal{A})\right)}\right\}<\infty$, that is the operator

$$
\begin{array}{ccc}
\mathcal{M}: A^{0}(G, \mathcal{A}) & \rightarrow & A^{0}(G, \mathcal{A}) \\
g & \mapsto & f g
\end{array}
$$

is completely bounded.

We have the following theorem which is a vector-valued extension of a result given by Gilbert [7] and proved by Jolissaint in [9].

Theorem 4.12 Let $G$ be a locally compact group, $\mathcal{A}$ a unital and commutative $H^{*}$-algebra and let $f \in C^{b}(G, \mathcal{A})$. The following assertions are equivalent:

(i) $f$ is a completely bounded multiplier on $A^{0}(G, \mathcal{A})$.

(ii) there exists an integer $n \in \mathbb{N}^{*}$, a family $\left(a_{i}\right)_{i \in I}$ with $a_{i} \in \mathcal{A}$, a Hilbert space $K$ and two families of bounded continuous functions $\left(\alpha_{i}\right)_{i \in I},\left(\beta_{i}\right)_{i \in I}$ from $G$ to $K$ such that for all $s, t \in G$,

$$
f\left(t^{-1} s\right)=\sum_{i \in I}\left(\left\langle\alpha_{i}(s), \beta_{i}(t)\right\rangle_{K}\right) a_{i}
$$

where $\langle\cdot, \cdot\rangle_{K}$ denotes the inner-product on $K$ and $I=\{1,2, \cdots, n\} \subset \mathbb{N}^{*}$.

\section{Proof.}

(i) $\Rightarrow$ (ii): Since $f \in C^{b_{0}}(G, \mathcal{A})$, there exist $n \in \mathbb{N}^{*}, a_{1}, a_{2}, \cdots, a_{n} \in \mathcal{A}$ and $f_{1}, f_{2}, \cdots, f_{n} \in A(G)$ such that

$$
f=\sum_{i=1}^{n} a_{i} f_{i}
$$

If $f=\sum_{i=1}^{n} a_{i} f_{i}$ is a completely bounded multiplier on $A^{0}(G, \mathcal{A})$, then for each $1 \leq i \leq n$, $f_{i}$ is a completely bounded multiplier on $A(G)$ (Lemma 4.11). Using Gilbert's Theorem, we claim that for each $i$, there exist a Hilbert space $K_{i}$ and two bounded continuous functions $\gamma_{i}, \delta_{i}$ from $G$ to $K_{i}$ such that

$$
f_{i}\left(t^{-1} s\right)=\left\langle\alpha_{i}(s), \beta_{i}(t)\right\rangle \text { for all } s, t \in G \text {. }
$$

Each $\gamma_{i}$ (resp. $\delta_{i}$ ) can be identified to the element

$$
\alpha_{i}=(0, \ldots, 0, \underbrace{\gamma_{i}}_{i^{t h} \underbrace{}_{\text {component }}}, 0, \ldots, 0)\left(\operatorname{resp} . \beta_{i}=(0, \ldots, 0, \underbrace{\left.\delta_{\text {component }}^{\delta_{i}}, 0, \ldots, 0\right)}_{i^{\text {th }}})\right.
$$

of the Hilbert space

$$
K=\bigoplus_{i \in I} K_{i}
$$

Finally,

$$
f\left(t^{-1} s\right)=\sum_{i=1}^{n}\left\langle\alpha_{i}(s), \beta_{i}(t)\right\rangle_{K} a_{i}
$$


(ii) $\Rightarrow$ (i): Conversely, assume

$$
f\left(t^{-1} s\right)=\sum_{i=1}^{n}\left\langle\alpha_{i}(s), \beta_{i}(t)\right\rangle_{K} a_{i}, \quad \text { then } f(\mathrm{t})=\sum_{i=1}^{n}\left(\left\langle\alpha_{i}(t), \beta_{i}\left(1_{G}\right)\right\rangle_{K}\right) a_{i} .
$$

Let $f_{i}$ be the functions from $G$ to $\mathbb{C}$ such that $f_{i}\left(t^{-1} s\right)=\left\langle\alpha_{i}(s), \beta_{i}(t)\right\rangle_{K}$ for all $s, t \in G$, thus $f_{i}: t \mapsto$ $\left\langle\alpha_{i}(t), \beta_{i}\left(1_{G}\right)\right\rangle_{K}$ are bounded on $G$ and we have

$$
f(t)=\sum_{i=1}^{n}(t) a_{i}
$$

Using Lemma 4.11, all we have to prove is that each $f_{i}$ is a completely bounded multiplier on $A(G)$. This is obvious according to Gilbert's Theorem.

\section{Group von Neumann algebras and operator spaces}

The group von Neumann algebra $V N(G)(\operatorname{resp} . V N(G, \mathcal{A}))$ is a closed subspace of $\mathcal{B}\left(L_{2}(G)\right)\left(\operatorname{resp} . \mathcal{B}\left(L_{2}(G, \mathcal{A})\right)\right.$ ) and then, is an operator space. Moreover, since $A(G)$ (resp. $A(G, \mathcal{A})$ ) is a predual of a von Neumann algebra, it can be equipped with its canonical operator space structure.

In this section, the $H^{*}$-algebra $\mathcal{A}$ is assumed to have a dual operator space structure, i.e. $\mathcal{A}$ is an operator space and there exists an operator space $E$ such that $\mathcal{A}$ is completely isometric to the dual operator $E^{*}$ of $E$. The operator space $E$ is called the operator predual of the dual operator space $\mathcal{A}$ and shall be denoted $\mathcal{A}_{*}$ (for more details about operator predual of a dual operator space, see [12]).

\section{Theorem 5.1}

(i) The space $V N(G) \bigotimes_{\min } \mathcal{A}$ is completely and isometrically isomorphic to the space $\operatorname{VN}(G, \mathcal{A})$.

(ii) We have the completely isometric injection

$$
A(G) \otimes_{\min } \mathcal{A} \hookrightarrow\left(V N(G) \widehat{\otimes} \mathcal{A}_{*}\right)^{*} .
$$

Proof. The proof of this theorem will be largely analogous to that of Grothendieck's theorem [8] (\$2, section 1 théorème 2).

(i) Consider the mapping $\mathcal{H}: V N(G) \otimes \mathcal{A} \rightarrow V N(G, \mathcal{A})$,

such that

$$
\begin{gathered}
(\mathcal{H} u)(t)=\sum_{k=1}^{m} u_{\mathrm{k}}(\mathrm{t}) a_{k} \\
\text { where } u=\sum_{k=1}^{m} u_{\mathrm{k}} \otimes a_{k} \in V N(G) \stackrel{\vee}{\otimes} \mathcal{A} \text { and } t \in G .
\end{gathered}
$$

$\mathcal{H} v$ is then an element of $C_{c}(G, \mathcal{A})$ equipped with the norm $\|\cdot\|_{\infty}$.

Let $n \in \mathbb{N}^{*}$, consider also the mapping

$$
\mathcal{H}_{n}: M_{n}(V N(G) \stackrel{\vee}{\otimes} \mathcal{A}) \rightarrow M_{n}(V N(G, \mathcal{A})),
$$

such that 


$$
\begin{gathered}
\left(\mathcal{H}_{n} v\right)(t)=\left(\left(\mathcal{H} v_{i j}\right)(t)\right)_{1 \leq i, j \leq n}=\left(\sum_{\mathrm{k}=1}^{\mathrm{m}} v_{i j}^{k}(\mathrm{t}) a_{i j}^{k}\right)_{1 \leq i, j \leq n} \\
\text { where } v=\left(v_{i j}\right)_{1 \leq i, j \leq n} \in M_{n}(V N(G) \stackrel{\vee}{\otimes} \mathcal{A}), \\
\text { with } v_{i j}=\sum_{k=1}^{m} v_{i j}^{k} \otimes a_{i j}^{k} \in V N(G) \otimes \mathcal{A} \text { and } t \in G .
\end{gathered}
$$

$\mathcal{H}_{n} v$ is then an element of $C_{c}\left(G, M_{n}(\mathcal{A})\right)$ equipped with the norm $\|\cdot\|_{\infty}$.

We have to prove that $\forall n \in \mathbb{N}^{*}, \mathcal{H}_{n}$ is an isometric isomorphism and we are done.

$$
\begin{aligned}
& \left\|\mathcal{H}_{n} v\right\|_{\infty}=\quad \sup \left\{\left\|\left(\sum_{k=1}^{m} v_{i j}^{k}(g) a_{i j}^{k}\right)_{1 \leq i, j \leq n}\right\|_{M_{n}(\mathcal{A})}: g \in G\right\} \\
& =\quad \sup \left\{\sup _{1 \leq i, j \leq n}\left\|\sum_{k=1}^{m} v_{i j}^{k}(g) a_{i j}^{k}\right\|_{\mathcal{A}}: g \in G\right\} \\
& =\sup \left\{\sup _{1 \leq i, j \leq n}\left|a^{*}\left(\sum_{k=1}^{m} v_{i j}^{k}(g) a_{i j}^{k}\right)\right|: g \in G, a^{*} \in \mathcal{A}^{*},\left\|a^{*}\right\| \leq 1\right\} \\
& =\sup \left\{\sup _{1 \leq i, j \leq n}\left|\sum_{k=1}^{m} v_{i j}^{k}(g) a^{*}\left(a_{i j}^{k}\right)\right|: g \in G, a^{*} \in \mathcal{A}^{*},\left\|a^{*}\right\| \leq 1\right\} \\
& =\sup \left\{\sup _{1 \leq i, j \leq n}\left\{\sup _{g \in G}\left|\sum_{k=1}^{m} a^{*}\left(a_{i j}^{k}\right) v_{i j}^{k}(g)\right|\right\}: a^{*} \in \mathcal{A}^{*},\left\|a^{*}\right\| \leq 1\right\} \\
& =\sup \left\{\sup _{1 \leq i, j \leq n}\left\|\sum_{k=1}^{m} a^{*}\left(a_{i j}^{k}\right) v_{i j}^{k}\right\|_{\infty}: a^{*} \in \mathcal{A}^{*},\left\|a^{*}\right\| \leq 1\right\} \\
& =\sup \left\{\sup _{1 \leq i, j \leq n}\left|\varpi\left(\sum_{k=1}^{m} a^{*}\left(a_{i j}^{k}\right) v_{i j}^{k}\right)\right|: \varpi \in V N(G)^{*}, a^{*} \in \mathcal{A}^{*},\|\varpi\| \leq 1,\left\|a^{*}\right\| \leq 1\right\} \\
& =\sup \left\{\sup _{1 \leq i, j \leq n}\left|\sum_{k=1}^{m} a^{*}\left(a_{i j}^{k}\right) \varpi\left(v_{i j}^{k}\right)\right|: \varpi \in V N(G)^{*}, a^{*} \in \mathcal{A}^{*},\|\varpi\| \leq 1,\left\|a^{*}\right\| \leq 1\right\} \\
& =\sup \left\{\sup _{1 \leq i, j \leq n}\left|\left(\varpi \otimes a^{*}\right)\left(\sum_{k=1}^{m} v_{i j}^{k} \otimes a_{i j}^{k}\right)\right|: \varpi \in V N(G)^{*}, a^{*} \in \mathcal{A}^{*},\|\varpi\| \leq 1,\left\|a^{*}\right\| \leq 1\right\} \\
& =\sup _{1 \leq i, j \leq n}\left\|\sum_{k=1}^{m} v_{i j}^{k} \otimes a_{i j}^{k}\right\|_{V} \\
& =\quad\|v\| \\
& \text { that is }\left\|\mathcal{H}_{n} v\right\|_{\infty}=\|v\| \text {. }
\end{aligned}
$$

We just proved that $M_{n}(V N(G) \stackrel{\vee}{\otimes} \mathcal{A})$ is isometrically isomorphic with the closed linear subspace of $M_{n}(V N(G, \mathcal{A})) \cong V N\left(G, M_{n}(\mathcal{A})\right)$ generated by the family of functions of the form 


$$
\begin{aligned}
G & \rightarrow \quad M_{n}(\mathcal{A}) \\
g & \mapsto\left(\sum_{k=1}^{m} v_{i j}^{k}(g) \otimes a_{i j}^{k}\right)
\end{aligned}
$$

where $v_{i j}^{k} \in V N(G)$ and $a_{i j}^{k} \in \mathcal{A}$ for $1 \leq i, j \leq n$ and $1 \leq k \leq m$; all we have left to do is to show that this family is dense in $\operatorname{VN}\left(G, M_{n}(\mathcal{A})\right)$.

Let $f_{n}: G \rightarrow M_{n}(\mathcal{A})$ be continuous and let $\varepsilon>0$ be given. $f_{n}(G)$ is compact so there are points $t_{1}, t_{2} \cdots, t_{m} \in G$ such that for any $t \in G$ there's a $\ell: 1 \leq \ell \leq m$ for which $\left\|f_{n}(t)-f_{n}\left(t_{\ell}\right)\right\| \leq \varepsilon / 2$, say. Let $U_{\ell}=\left\{t:\left\|f_{n}(t)-f_{n}\left(t_{\ell}\right)\right\| \leq \varepsilon\right\}$. Then $\left\{U_{1}, \cdots, U_{m}\right\}$ is a finite open cover of $G$ and therefore, there is a continuous partition of unity $\left\{f_{n 1}, f_{n 2} \cdots, f_{n m}\right\}$ subordinate to $\left\{U_{1}, \cdots, U_{m}\right\}$, that is, there are continuous real-valued functions $f_{n 1}, f_{n 2} \cdots, f_{n m}$ on $G$ each having values in $[0,1]$ with

Define $h_{n}: G \rightarrow M_{n}(A)$ by

$$
\sum_{k=1}^{m} f_{n k}(t) \equiv I_{n} \text { and } f_{n k}(t)=0 \text {, when } t \text { is outside } U_{k} \text {. }
$$

$$
\begin{aligned}
& h_{n}(t)=\sum_{\ell=1}^{m} f_{n \ell}(t) f_{n}\left(t_{\ell}\right) \\
& \text { Plainly } t=\mathcal{H}_{n}\left(\sum_{\ell=1}^{m} f_{n \ell} \otimes f_{n}\left(t_{\ell}\right)\right)
\end{aligned}
$$

and if $t \in G$, then

$$
\begin{aligned}
\left\|h_{n}(t)-f_{n}(t)\right\| & =\left\|\sum_{\ell=1}^{m} f_{n \ell}(t) f_{n}\left(t_{\ell}\right)-f_{n}(t)\right\| \\
& =\left\|\sum_{\ell=1}^{m} f_{n \ell}(t)\left[f_{n}\left(t_{\ell}\right)-f_{n}(t)\right]\right\| \\
& =\left\|\sum_{\ell: t \in U_{\ell}} f_{n \ell}(t)\left[f_{n}\left(t_{\ell}\right)-f_{n}(t)\right]\right\| \\
& <\varepsilon,
\end{aligned}
$$

it follows that $\left\|h_{n}-f_{n}\right\|_{\infty} \leq \varepsilon$ and with this the density of $\mathcal{H}_{n}{ }^{\prime}$ range is plain.

(ii) Since for any operator space $X$ and $Y$, the natural embedding

$X^{*} \bigotimes_{\min } Y \hookrightarrow c b(X, Y)$ is completely isometric and we have the complete isometries $(X \widehat{\otimes} Y)^{*} \cong c b\left(X, Y^{*}\right) \cong c b\left(Y, X^{*}\right)$ (Corollary 7.1.5 and Proposition 8.1.2 in [4]), we have:

$A(G) \otimes_{\min } \mathcal{A} \hookrightarrow \operatorname{cb}(V N(G), \mathcal{A}) \cong\left(V N(G) \widehat{\otimes} \mathcal{A}_{*}\right)^{*}$ 


\section{References}

[1] W. Ambrose, Structure theorems for a special class of Banach algebras. Trans. Amer. Math. Soc. 57, 364-386 (1945).

[2] D. Blecher and V. Paulsen, Tensor products of operator's spaces. J. Funct. Anal. 99, 262-292 (1991).

[3] J. Diestel, J. H. Fourie and J. Swart, The Metric Theory of Tensor Products, Grothendieck's Résumé Revisited. Amer. Math. Soc. (2008).

[4] E. Effros and Z. J. Ruan, Operator spaces., Oxford University Press Inc., New York (2000, reprinted 2005)

[5] E. Effros and Z. J. Ruan, On the abstract characterization of operator spaces. Proc. Amer. Math. Soc. 119, 579584 (1993).

[6] P. Eymard, L'algèbre de Fourier d'un groupe localement compact, Bull. Soc. Math. France 92, 181-236 (1964).

[7] J. E. Gilbert, $L_{p}$-convolution operators and tensor products of Banach spaces, I, II, III, preprints.

[8] A. Grothendieck, Résumé de la théorie métrique des produits tensoriels topologiques.Bol. Soc. Mat. Sao Paulo, 8, 1-79 (1953/1956).

[9] P. Jolissaint, A characterization of completely bounded multipliers of Fourier algebras, Colloq. Math. 63 311313 (1992).

[10] F. J. Murray and J. v. Neumann, On rings of operators, Annals of Mathematics Second Series, 37, 1, 116-229 (1936).

[11] V. Paulsen, Completely bounded maps and operator algebras, Cambridge, Cambridge, UK, (2002).

[12] Z.J. Ruan, On the predual of dual algebras. J. Operator Theory, 27,179-192 (1992).

[13] D. Z. Spicer, Group algebras of vector valued functions. Pacific Journal of Mathematics24, 2,379-399(1968).

[14] W. Stinespring, Integration theorem for gages and duality for unimodular groups. Trans. Amer. Math. Soc.90, 15-26 (1959). 